\title{
Poor Renal and Cardiovascular Outcomes in Patients with Biopsy-Proven Diabetic Nephropathy
}

\author{
Yiyun Wang Ting Zhou Qiming Zhang Yang Fei Ze Li Shiqi Li \\ Li He Qunzi Zhang Yang Dong Ying Fan Niansong Wang
}

Department of Nephrology, Shanghai Jiao Tong University Affiliated Sixth People's Hospital, Shanghai, China

\section{Keywords}

Renal biopsy · Diabetic nephropathy $\cdot$ Non-diabetic renal disease $\cdot$ Renal prognosis

Cardiovascular prognosis

\begin{abstract}
Background: Despite the high mortality of cardiovascular disease (CVD) in diabetic patients with renal injury, few studies have compared cardiovascular characteristics and outcomes between patients with diabetic nephropathy (DN) and non-diabetic renal disease (NDRD). Methods: A total of 326 type 2 diabetes mellitus patients with renal biopsy were assigned to DN and NDRD groups. Echocardiography and Doppler ultrasound were performed to evaluate left ventricular hypertrophy (LVH) and peripheral atherosclerosis disease (PAD). Renal and cardiovascular survival rates were compared between the DN and NDRD groups by Kaplan-Meier analysis. Risk factors for renal and cardiovascular events in DN patients were identified by a Cox proportional hazards model. Results: In total, 179 patients entered the DN group (54.9\%) and 147 made up the NDRD group (45.1\%). The presence of diabetic retinopathy, family history of diabetes, and dependence on insulin therapy were associated with the presence of DN. DN patients had more CVD with more severe LVH and PAD. Poorer renal (log-rank $\left.\chi^{2}=26.534, p<0.001\right)$ and cardiovascular (log-rank $\left.\chi^{2}=16.257, p<0.001\right)$ prognoses were seen in the DN group. DR (HR 1.539, 95\% Cl 1.332-1.842), eGFR (HR 0.943, 95\% Cl 0.919-0.961), and $24-\mathrm{h}$ proteinuria (HR 1.211,95\% Cl 1.132-1.387) were identified as risk factors for renal endpoints. Age (HR 1.672, 95\% Cl 1.487-1.821), HbA1C (HR 1.398, 95\% Cl 1.197-1.876), and 24-h proteinuria (HR 1.453, 95\% Cl 1.289-1.672) were associated with cardiovascular endpoints. Conclusion: Patients with DN had more severe CVD along with poorer renal and cardiovascular prognoses than those with NDRD.

Y.W. and T.Z. contributed equally to this study. 


\section{Kidney \\ Blood Pressure \\ Research}

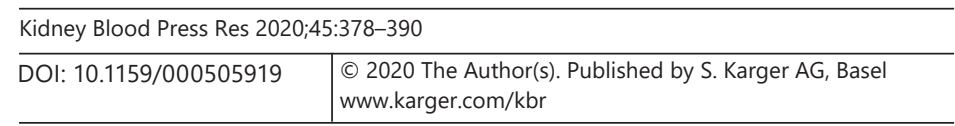

Wang et al.: Poor Prognosis of Biopsy-Proven Diabetic Nephropathy

\section{Introduction}

Rapid economic growth and related lifestyle changes are associated with an alarming increase in the prevalence of diabetes mellitus (DM). Diabetic nephropathy (DN) is one of the most common complications of DM and is the leading cause of end-stage renal disease in many developed countries [1]. In China, the incidence of chronic kidney disease related to DM has exceeded that of glomerulonephritis since 2011 [2]. Therefore, identifying individuals at high risk of developing DN can enable early, targeted intervention to prevent renal function decline and to improve patient outcomes.

In clinical practice, renal biopsy is not routinely performed in diabetic patients with renal injury. However, studies have shown that non-diabetic renal disease (NDRD) is also common in patients with type 2 DM (T2DM), alone or with underlying DN [3-12]. Since a significant proportion of diabetic patients with renal injury are diagnosed clinically as DN without pathological confirmation, those with NDRD are likely to be misdiagnosed as DN. As some types of NDRD, such as membranous nephropathy (MN) and IgA nephropathy (IgAN), are potentially treatable with renin-angiotensin-aldosterone system (RAAS) inhibitors and immunosuppressive therapy, renal biopsy would allow a precise diagnosis to be made and enable appropriate treatment and better prognosis. Many studies have evaluated the pathological profile of renal diseases in diabetic patients along with the identification of clinical predictors for DN. However, the results of these studies have been inconsistent, possibly due to different biopsy criteria and races [3-12]. In addition, few studies have performed long-term follow-up $[3,7,11]$, and the prognosis remained unclear in different renal diseases among diabetic patients.

In addition to renal impairment, many diabetic patients also suffer from various cardiovascular diseases (CVD) [13]. As the most common cause of mortality in diabetic patients, CVD accounts for $52 \%$ of deaths in patients with T2DM and $44 \%$ in patients with type $1 \mathrm{DM}$ (T1DM) [14]. Interestingly, several recent studies have confirmed the connection between diabetic microvascular disease and macrovascular disease. The link is most obvious for patients with DN, which has been identified as a major predictor of cardiovascular morbidity and mortality [15-18]. Left ventricular hypertrophy (LVH), a major manifestation of diabetic cardiomyopathy [19], has been identified as an independent risk factor for myocardial infarction, heart failure, arrhythmia, and other causes of cardiovascular deaths [20, 21]. In addition to $\mathrm{LVH}$, peripheral atherosclerosis disease (PAD) is also a well-known risk factor for cardiovascular and cerebrovascular ischemic events, affecting both the quality of life and life expectancy [22-25]. Therefore, LVH and PAD may serve as early indicators of cardiovascular damage among diabetic patients with renal injury. However, the incidence and severity of LVH and PAD were still unclear in biopsy-proven DN patients and the differences of these two indicators between DN and NDRD patients were also obscure.

In the current study, we aimed to evaluate biopsy-proven renal disease among diabetic patients and to identify clinical predictors of DN. In addition, we compared the incidence and severity of LVH and PAD between patients with DN and NDRD to identify any differences in early cardiovascular changes. In addition, we also explored how much the known effects of DM on CV changes (LVH and atherosclerosis) are actually dependent on the reduced renal function. Follow-up data were analyzed to compare renal and cardiovascular survival and to identify risk factors for both renal and cardiovascular events. 


\section{Kidney \\ Blood Pressure \\ Research}

\begin{tabular}{l|l}
\hline Kidney Blood Press Res 2020;45:378-390 \\
\hline DOI: 10.1159/000505919 & $\begin{array}{l}\text { @ 2020 The Author(s). Published by S. Karger AG, Basel } \\
\text { www.karger.com/kbr }\end{array}$ \\
\hline
\end{tabular}

Wang et al.: Poor Prognosis of Biopsy-Proven Diabetic Nephropathy

\section{Materials and Methods}

\section{Study Design and Participants}

Data from patients with T2DM who underwent renal biopsy at the Shanghai Sixth People's hospital between January 2008 and July 2019 were retrospectively reviewed. Eligible patients were categorized into two groups: DN and NDRD group. Baseline clinical and laboratory characteristics were compared among the groups. Patients with incomplete data, unclear medical history, extremely high ( $>180 / 110 \mathrm{~mm} \mathrm{Hg}$ ) and low ( $<90 / 60 \mathrm{~mm} \mathrm{Hg}$ ) blood pressure, renal biopsy material including fewer than five glomeruli, and severe infection or malignancy were excluded from the study (online suppl. Fig. S1; for all online suppl. material, see www.karger. com/doi/10.1159/000505919).

The study protocol was approved by the Ethics Committee of the Shanghai Jiao Tong University Affiliated Sixth People's Hospital and was conducted in full accordance with the principles of the declaration of Helsinki. All patients provided written informed consent.

\section{Clinical Data and Laboratory Results}

The following clinical parameters were collected: sex, age, course of DM, family history of DM, use of OHD (oral hypoglycemic drugs) and insulin, presence of diabetic retinopathy (DR), presence of hypertension, systolic blood pressure, diastolic blood pressure, and use of RAAS inhibitors.

The following laboratory indicators were measured by standard laboratory methods: fasting plasma glucose, 2-h postprandial plasma glucose, glycosylated hemoglobin A1c (HbA1c), serum creatinine ( $\mathrm{sCr}$ ), blood urea nitrogen, uric acid, estimated glomerular filtration rate (eGFR; calculated by the MDRD formula), presence of glomerular hematuria, 24-h urinary protein, hemoglobin, serum albumin, serum calcium, serum phosphorus, total triglycerides, total cholesterol, high-density lipoprotein cholesterol, and low-density lipoprotein cholesterol.

\section{Renal Biopsy and Pathological Classification}

From a total of 452 T2DM patients who underwent renal biopsy during the study period, 326 were eligible for enrollment. Renal biopsies were performed for the following indications: (1) proteinuria over $1 \mathrm{~g} / 24 \mathrm{~h}$ and diabetic duration less than 5 years; (2) presence of renal damage without DR; (3) rapid decline in eGFR within 3 months; (4) presence of microscopic glomerular hematuria. Biopsy samples were cut into 3- $\mu$ m sections and stained with hematoxylin and eosin, periodic acid-Schiff, and Masson trichrome. All specimens were processed for electron microscopy and reviewed independently by 2 pathologists. A diagnosis of DN was made according to the 2010 DN classification [26].

\section{Echocardiography}

Tracings were taken with patients in a partial left decubitus position, using a Vingmed System 7 ultrasound machine (Philips, Germany). Left atrial diameter (LAD), left ventricular end-diastolic dimension (LVDD), left ventricular end-systolic dimension, interventricular septal thickness (IVST), and posterior wall end-diastolic thickness were measured in accordance with the guidelines of the American Society of Echocardiography [27]. Early (E) and late (A) diastolic velocities, and ratios of early and late velocities (E/A), were also determined.

LAD $>30 \mathrm{~mm}$ in women and $>33 \mathrm{~mm}$ in men was defined as left atrial enlargement (LAE). LVDD $>50 \mathrm{~mm}$ in women and $>55 \mathrm{~mm}$ in men was defined as left ventricular enlargement. Left ventricular mass (LVM) was calculated using the Devereux formula [28]. LVM index (LVMI) was derived by correcting LVM for BSA [29]. LVH was defined as follows: LVMI >115 $\mathrm{g} / \mathrm{m}^{2}$ for men and LVMI $>95 \mathrm{~g} / \mathrm{m}^{2}$ for women [30]. 


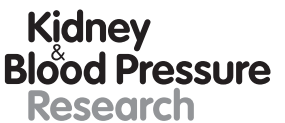

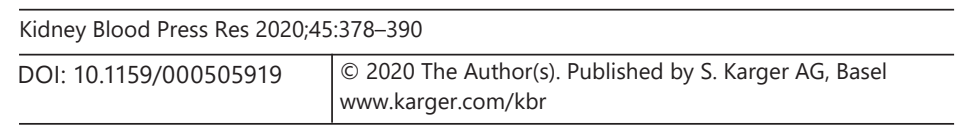

Wang et al.: Poor Prognosis of Biopsy-Proven Diabetic Nephropathy

\section{Doppler Ultrasonography Measurements}

Doppler ultrasound examination (Siemens; 5- to 13-MHz linear array probe) was performed to detect atherosclerotic lesions in both carotid arteries and lower limb arteries, including measurement of the intima-media thickness (IMT), atherosclerotic plaques, and stenosis. In accordance with the consensus of Mannheim, atherosclerotic plaques were defined as a local structural invasion of the artery cavity of $0.5 \mathrm{~mm}$ or $50 \%$, with a thickness of $>1.5 \mathrm{~mm}$. Plaques with low echogenicity in $>50 \%$ of their area were defined as unstable [31].

\section{Follow-Up and Endpoints}

Renal endpoints were defined as a doubling of baseline $\mathrm{sCr}$, initiation of renal replacement therapy, or death. Cardiovascular endpoints were defined as stroke, transient ischemic attack, unstable angina, myocardial infarction, hospitalization caused by acute heart failure, requirement for revascularization of coronary or lower extremity arteries, or death [32].

\section{Statistical Analysis}

Statistical analyses were performed using SPSS software (version 25.0; SPSS Inc., Chicago, IL, USA). Categorical data were expressed as the frequency (\%), normally distributed data as the mean ( \pm standard deviation, SD), and skewed data as the median with an interquartile range. The proportions were compared using the $\chi^{2}$ test. Comparisons of continuous variables between the groups were performed using the independent $t$ test for normally distributed data and the Mann-Whitney U test for data not normally distributed. Multivariate logistic regression analyses were performed to identify clinical risk factors for DN. Cumulative renal and cardiovascular survival was estimated using Kaplan-Meier survival curves and compared using the log-rank test. A multivariate Cox proportional hazards regression model was used to identify factors that significantly affected renal and cardiovascular outcomes in diabetic patients with renal injury in DN patients.

\section{Results}

\section{Clinical Characteristics and Laboratory Findings}

Of 452 T2DM patients who underwent renal biopsy during the study period, 326 were eligible for enrollment: 179 (54.9\%) in the DN group and 147 (45.1\%) in the NDRD group. Baseline patient characteristics are summarized in Table 1. Patients in the DN group were prone to have family diabetic history, use of insulin, and DR. Other baseline characteristics, including age, gender, diabetic duration, use of OHD and RAAS inhibitors, control of blood glucose and pressure, renal function, proteinuria, hematuria, and blood lipid level, were not significantly different.

A total of 296 patients underwent fundoscopy (164 in the DN group, 132 in the NDRD group). We further conducted a sub-analysis to clarify the differences of baseline characteristics by dividing DN patients into two groups: DN without DR $(n=128)$ and DN with DR $(n=$ 36; online suppl. Table S1). Patients with both DN and DR had more familial history of diabetes, a longer diabetic duration, use of insulin, and more prevalent hypertension. They also had poorer renal function with significantly lower eGFR and higher levels of UACR and more prominent proteinuria when compared to DN patients without DR. Dyslipidemia was also more prevalent in patients with both DN and DR. 
Table 1. Baseline characteristics of the study population

\begin{tabular}{|c|c|c|c|}
\hline & $\operatorname{NDRD}(n=147)$ & $\mathrm{DN}(n=179)$ & $p$ value \\
\hline Age, years & $56.14 \pm 12.35$ & $54.16 \pm 10.72$ & 0.123 \\
\hline Sex, male & 111 (75.5) & 143 (77.9) & 0.343 \\
\hline Course of DM, years & $7.37 \pm 2.46$ & $7.50 \pm 2.64$ & 0.204 \\
\hline Family history of DM & $101(68.7)$ & $156(87.2)^{*}$ & $<0.001$ \\
\hline Use of OHD & $111(75.5)$ & $124(69.3)$ & 0.212 \\
\hline Use of insulin & $90(61.2)$ & $140(78.2)^{*}$ & 0.001 \\
\hline DR & $61(27.1)$ & $128(71.5)^{*}$ & $<0.001$ \\
\hline Hypertension & $117(79.6)$ & $164(91.6)^{*}$ & 0.002 \\
\hline SBP, mm Hg & 137 (123-150) & $140(125-156)$ & 0.140 \\
\hline DBP, mm Hg & $82.16 \pm 11.59$ & $83.05 \pm 11.87$ & 0.498 \\
\hline RAAS inhibitor & $105(71.4)$ & $133(74.3)$ & 0.561 \\
\hline $\mathrm{FPG}, \mathrm{mmol} / \mathrm{L}$ & $8.11 \pm 2.61$ & $8.29 \pm 2.89$ & 0.551 \\
\hline 2h-PPG, mmol/L & $11.77 \pm 4.00$ & $11.84 \pm 4.02$ & 0.880 \\
\hline $\mathrm{HbA1c}, \%$ & $7.71 \pm 1.49$ & $7.76 \pm 1.59$ & 0.076 \\
\hline $\mathrm{sCr}, \mu \mathrm{mol} / \mathrm{L}$ & $107.19 \pm 76.67$ & $110.74 \pm 68.25$ & 0.875 \\
\hline BUN, mmol/L & $7.70(6.21-9.81)$ & $7.61(6.02-9.01)$ & 0.906 \\
\hline Uric acid, umol/L & $365.43 \pm 83.55$ & $373.55 \pm 87.65$ & 0.825 \\
\hline $\mathrm{eGFR}, \mathrm{mL} / \mathrm{min} / 1.73 \mathrm{~m}^{2}$ & $66.28 \pm 30.92$ & $70.95 \pm 34.24$ & 0.202 \\
\hline Hematuria & $95(64.6)$ & $104(58.1)$ & 0.229 \\
\hline 24-h proU, g & $3.42(2.20-5.98)$ & $3.46(1.89-6.09)$ & 0.517 \\
\hline Hemoglobin, g/L & $119(108-136)$ & $120(98-132)$ & 0.810 \\
\hline Albumin, g/L & $37.20 \pm 6.98$ & $36.98 \pm 7.03$ & 0.781 \\
\hline Calcium, mmol/L & $2.23 \pm 0.14$ & $2.21 \pm 0.23$ & 0.966 \\
\hline Phosphorus, mmol/L & $1.18 \pm 0.22$ & $1.19 \pm 0.23$ & 0.928 \\
\hline $\mathrm{TC}, \mathrm{mmol} / \mathrm{L}$ & $5.32 \pm 1.67$ & $5.40 \pm 1.83$ & 0.664 \\
\hline $\mathrm{TG}, \mathrm{mmol} / \mathrm{L}$ & $2.08 \pm 1.56$ & $2.17 \pm 1.75$ & 0.573 \\
\hline HDL-C, mmol/L & $1.08 \pm 0.27$ & $1.04 \pm 0.27$ & 0.168 \\
\hline LDL-C, mmol/L & $3.17 \pm 1.29$ & $3.22 \pm 1.43$ & 0.733 \\
\hline
\end{tabular}

Data are presented as $n(\%)$, the mean \pm SD, or median (IQR). ${ }^{*} p<0.05$ was considered significant compared with the NDRD group. DM, diabetes mellitus; OHD, oral hypoglycemic drugs; DR, diabetic retinopathy; SBP, systolic blood pressure; DBP, diastolic blood pressure; FPG, fasting plasma glucose; $2 \mathrm{~h}$ PPG, 2-h postprandial plasma glucose; HbA1c, glycosylated hemoglobin A1c; sCr, serum creatinine; BUN, blood urea nitrogen; eGFR, estimated glomerular filtration; 24-h proU, 24-h proteinuria; TC, total cholesterol; TG, triglycerides; HDL-C, high-density lipoprotein cholesterol; LDL-C, low-density lipoprotein cholesterol.

\section{Pathologic Diagnosis of Nephropathy}

The distribution of kidney disease in the NDRD group is shown in Figure 1. Of 147 patients with NDRD, 41 (27.9\%) had MN, 39 (26.5\%) had IgAN, and 33 (22.4\%) had mesangial proliferative glomerulonephritis (MsPGN). Hypertensive renal disease, focal segmental glomerulosclerosis, and Henoch-Schönlein purpura nephritis accounted for 4.8, 4.1, and 2.7\% of the NDRD group, respectively. Representative examples of DN and NDRD of histological images are show in Figure 2.

The quantification of pathological findings in DN patients are demonstrated in online supplementary Table S2. According to glomerular classification, 179 DN patients were grouped into classes I ( $n=11)$, IIa $(n=41)$, IIb $(n=52)$, III $(n=53)$, and IV $(n=22)$, respectively. In addition, most patients already had moderate to severe tubular damage at the time of biopsy with IFTA (interstitial fibrosis and tubular atrophy) scores of 2 and 3 observed in $59(33.0 \%)$ and $58(32.4 \%)$ patients, respectively. Severe vascular lesions were also identified with arteriolar hyalinosis of score 2 in 143 patients. 


\section{Kidney \\ Blood Pressure Research}

Fig. 1. Distribution of renal disease in the NDRD group. MN, membranous nephropathy; IgAN, IgA nephropathy; MsPGN, mesangial proliferative glomerulonephritis; HTRD, hypertensive renal disease; FSGS, focal segmental glomerular sclerosis; HSPN, Henoch-Schönlein purpura nephritis; LCDD, light chain deposition disease; MCD, minimal change disease; AAV, ANCA-associated vasculitis; LN, lupus nephritis; IgMN, IgM nephropathy; ATN, acute tubular necrosis; MPGN, membranous proliferative glomerulonephritis; TBMN, thin basement membrane nephropathy; CGN, crescentic glomerulonephritis; HBV-GN, HBV-associated nephritis.

\begin{tabular}{l|l}
\hline Kidney Blood Press Res 2020;45:378-390 \\
\hline DOI: 10.1159/000505919 & $\begin{array}{l}\text { @ 2020 The Author(s). Published by S. Karger AG, Basel } \\
\text { www.karger.com/kbr }\end{array}$ \\
\hline
\end{tabular}

Wang et al.: Poor Prognosis of Biopsy-Proven Diabetic Nephropathy

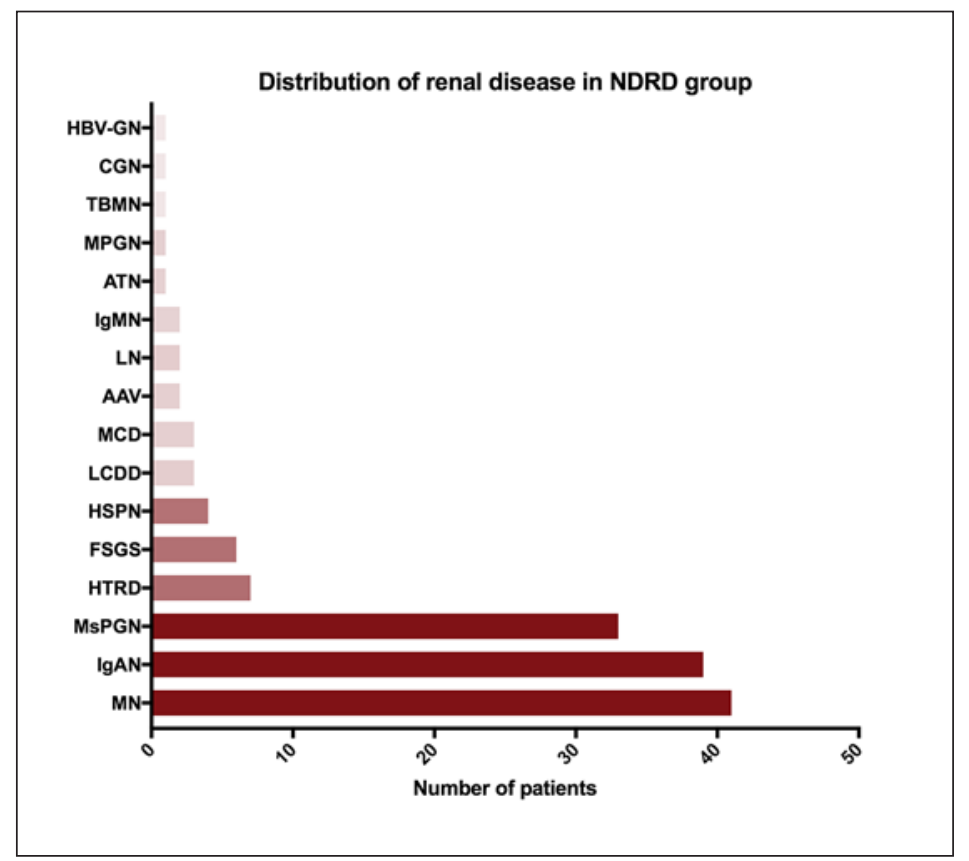

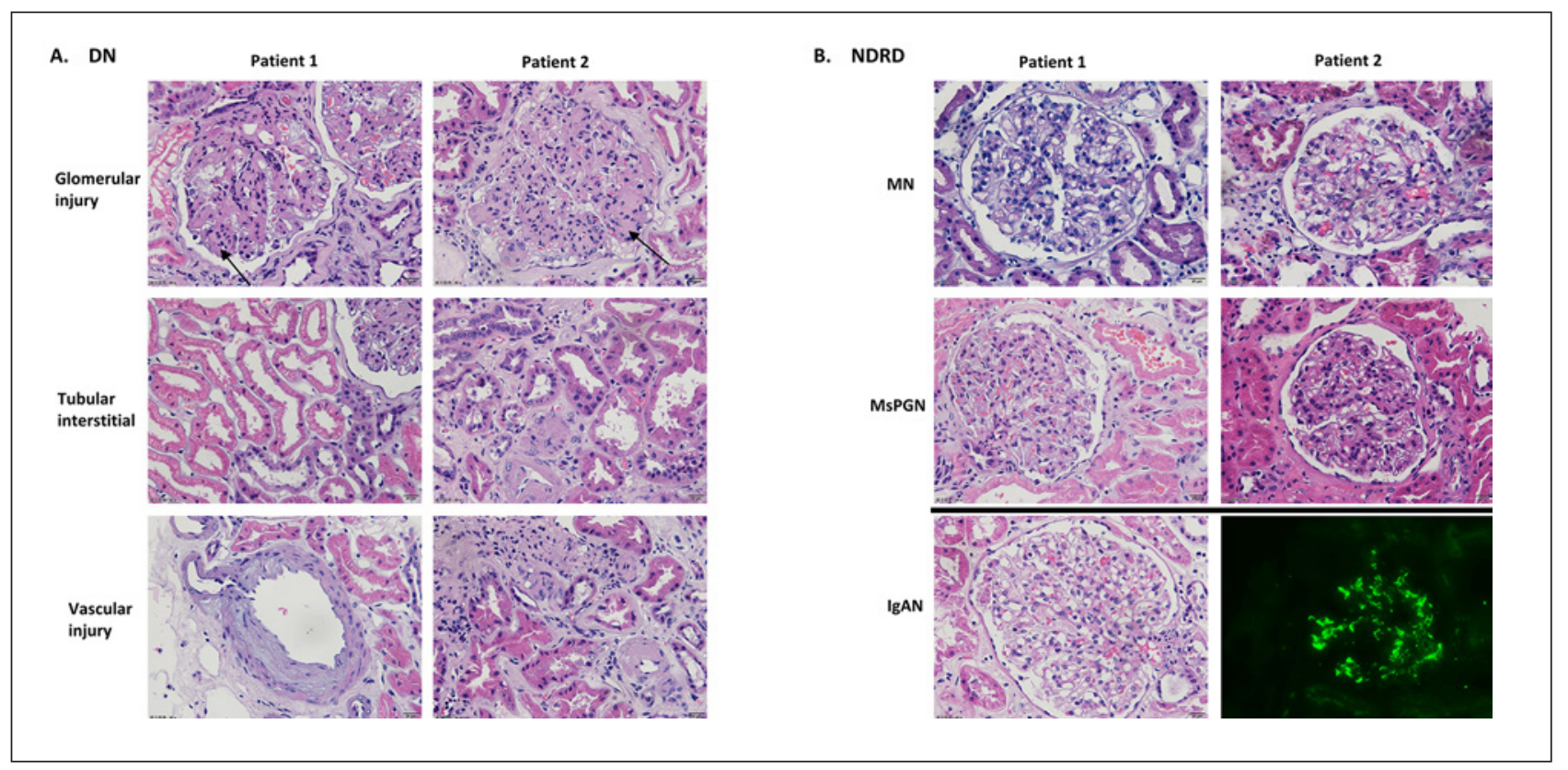

Fig. 2. Histological figures of DN (A) and NDRD (B) patients, each from 2 different patients. Images of DN, MN, MsPGN, and the first IgAN image are seen on light microscopy with periodic acid-Schiff reaction. Original magnification, $\times 400$. The final IgAN image is immunofluorescence.

\section{Risk Factors Associated with the Progression of DN}

To identify clinical indicators for the differences between DN and NDRD, a multivariate logistic regression analysis was conducted, and the results are shown in Table 2. After adjusting for baseline characteristics and laboratory results, three clinical parameters were identified as independent risk factors for biopsy-proven DN. Patients with DR appeared to be 
Table 2. Risk factors for DN identified by multivariate logistic regression analysis

\begin{tabular}{lllrrr}
\hline Indicators & $\beta$-Estimate & $\begin{array}{l}\text { Standard } \\
\text { error }\end{array}$ & $p$ value & OR & $95 \%$ CI \\
\hline Family history of DM (yes vs. no) & 1.248 & 0.374 & 0.001 & 3.485 & $\begin{array}{c}1.676-7.247 \\
\text { DR (yes vs. no) }\end{array}$ \\
Use of insulin (yes vs. no) & 1.338 & 0.434 & $<0.001$ & 20.609 & $\begin{array}{c}12.647-69.307 \\
1.579-5.660\end{array}$ \\
\hline
\end{tabular}

Multivariate logistic regression analysis was used to determine risk factors related to the presence of DN, and variables found statistically significant in univariate analysis were included in the multivariate logistic analysis. DR, diabetic retinopathy; OR, odds ratio.

Table 3. Comparison of echocardiographic characteristics and atherosclerotic lesions

\begin{tabular}{lcrr}
\hline & NDRD group & DN group & $p$ value \\
\hline Echocardiographic characteristics & & & 0.102 \\
LAD, mm & $39.42 \pm 3.80$ & $40.12 \pm 3.94$ & $<0.001$ \\
LAE & $107(72.8)$ & $165(92.2)^{*}$ & 0.074 \\
LVDD, mm & $45.27 \pm 3.76$ & $45.69 \pm 3.68$ & 0.938 \\
LVDS, mm & $30.44 \pm 3.70$ & $30.47 \pm 3.87$ & 0.675 \\
LVE & $10(6.8)$ & $17(9.5)$ & 0.022 \\
IVST, mm & $9.93 \pm 1.03$ & $10.21 \pm 1.16^{*}$ & 0.082 \\
PWTD, mm & $9.39 \pm 1.19$ & $9.65 \pm 1.46$ & 0.074 \\
LVEF, \% & $62.80 \pm 3.24$ & $62.92 \pm 3.21$ & 0.330 \\
E/A $<1$ & $72(49.0)$ & $78(43.6)$ & $<0.001$ \\
LVMI, g/m ${ }^{2}$ & $96.37 \pm 10.21$ & $101.20 \pm 11.68^{*}$ & 0.037 \\
LVH & $28(19)$ & $52(29.1)^{*}$ & 0.010 \\
\hline Atherosclerotic lesions & & & $<0.001$ \\
Mean CIMT, mm & $0.85 \pm 0.12$ & $0.89 \pm 0.14^{*}$ & 0.472 \\
Prevalence of carotid plaque & $50(34.0)$ & $119(66.5)^{*}$ & 0.195 \\
Prevalence of unstable carotid plaque & $37(25.2)$ & $60(33.5)$ & $<0.001$ \\
Prevalence of carotid stenosis & $7(4.8)$ & $15(8.4)$ & $<0.001$ \\
Mean FIMT, mm & $0.85 \pm 0.12$ & $0.92 \pm 0.13^{*}$ & 0.003 \\
Prevalence of plaque in LEA & $53(36.1)$ & $139(77.7)^{*}$ & 0.683 \\
Prevalence of unstable plaque in LEA & $13(8.8)$ & $37(20.7)^{*}$ & $13(7.3)$ \\
Prevalence of stenosis in LEA & $9(6.1)$ & & \\
\hline
\end{tabular}

Data are presented as $n(\%)$ or the mean \pm SD. $* p<0.05$ was considered significant compared with the NDRD group. LAD, left atrial dimension; LAE, left atrial enlargement; LVDD, left ventricular end-diastolic dimension; LVDS, left ventricular end-systolic dimension; LVE, left ventricular enlargement; IVST, interventricular septal thickness; PWTD, posterior wall end-diastolic thickness; LVEF, left ventricular ejection fraction; LVMI, left ventricular mass index; LVH, left ventricular hypertrophy; CIMT, carotid intima-medium thickness; FIMT, femoral intima-medium thickness; LEA, lower extremity arteries.

at highest risk of developing DN (OR 20.609; 95\% CI 12.647-69.307; $p<0.001$ ). In addition, patients with a family history of diabetes also showed a higher probability of DN (OR 3.485; 95\% CI 1.676-7.247; $p=0.001$ ), as did patients treated with insulin therapy (OR 2.989;95\% CI 1.579-5.660; $p=0.001)$.

\section{Comparison of Echocardiographic Parameters}

Echocardiography was conducted to compare cardiac parameters. When compared with patients in the NDRD group, those in the DN group showed thicker IVST (10.21 vs. $9.93 \mathrm{~mm}$, 


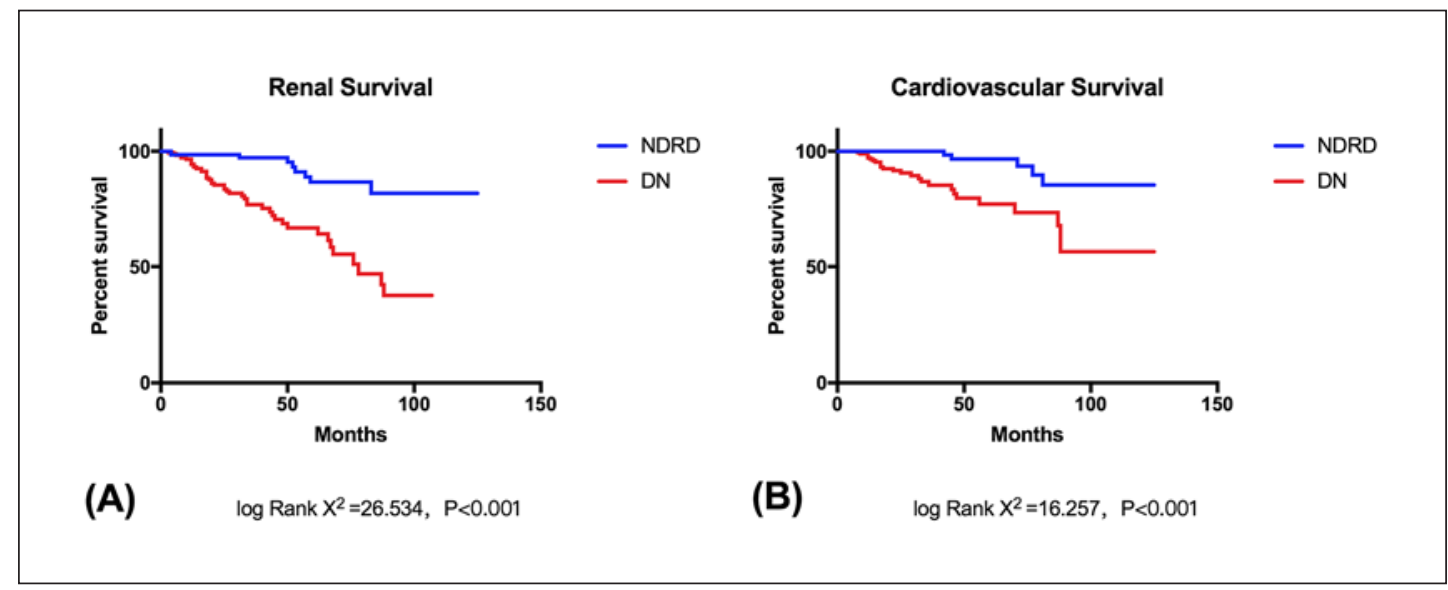

Fig. 3. Kaplan-Meier analysis for renal and cardiovascular survival. A Comparison of renal prognosis between DN and NDRD patients. B Comparison of cardiovascular prognosis between DN and NDRD patients. Differences between the groups were compared using the log-rank test.

$p=0.022)$, and LVMI (101.20 vs. $\left.96.37 \mathrm{~g} / \mathrm{m}^{2}, p<0.001\right)$. A higher incidence of LAE (92.2 vs. $72.8 \%, p<0.001)$, and LVH (29.1 vs. $19.0 \%, p=0.037$ ) was also found among patients with DN (Table 3). These data suggested that DN patients were more vulnerable to developing LVH than NDRD patients.

\section{Comparison of Peripheral Artery Atherosclerotic Lesions by Doppler Ultrasound}

Echo-Doppler of both carotid arteries and the lower extremity arteries was performed to measure the IMT, atherosclerotic plaques, and vascular stenosis. Both carotid IMT 0.89 vs. $0.85 \mathrm{~mm}, p=0.010$ ) and femoral IMT (0.92 vs. $0.85 \mathrm{~mm}, p<0.001$ ) were markedly thicker in the DN group (Table 3). Scatterplots of eGFR versus IMT in both the DN and NDRD groups are shown in online supplementary Figure S2. DN patients were also more likely to develop atherosclerotic plaques ( 66.5 vs. $34.0 \%$ in the carotid arteries and $77.7 \mathrm{vs.} 36.1 \%$ in the lower limb arteries, $p<0.001)$ and unstable plaques in the lower limb arteries (20.7 vs. 8.8\%, $p=$ 0.003). Therefore, DN patients tended to have more severe atherosclerotic lesions in peripheral arteries compared with the NDRD group.

\section{Comparison of Renal and Cardiovascular Survival}

Renal and cardiovascular outcomes were assessed in 165 DN and 134 NDRD patients with a medium follow-up period of 35 months, and details of renal and cardiovascular events are shown in online supplementary Table S3. Kaplan-Meier analysis showed that DN patients had poorer renal (log-rank $\chi^{2}=26.534, p<0.001$ ) and cardiovascular outcomes (log-rank $\chi^{2}=16.257, p<0.001$; Fig. 3 ). The Cox proportional hazards regression model of the DN group showed that DR (HR 1.539, 95\% CI 1.332-1.842), eGFR (HR 0.943, 95\% CI 0.919-0.961), and 24-h proteinuria (HR 1.211, 95\% CI 1.132-1.387) remained significantly associated with renal endpoints. Age (HR 1.672, 95\% CI 1.487-1.821), HbA1C (HR 1.398, 95\% CI 1.1971.876), 24-h proteinuria (HR 1.453, 95\% CI 1.289-1.672), and phosphorus (HR 1.021, 95\% CI 1.001-1.052) were identified as risk factors for cardiovascular endpoints (Table 4). Therefore, patients with DN tended to have poorer renal and cardiovascular outcomes than the NDRD group. 
Wang et al.: Poor Prognosis of Biopsy-Proven Diabetic Nephropathy

Table 4. Parameters identified by multivariate Cox proportional hazards regression analysis associated with renal and cardiovascular events in the DN group

\begin{tabular}{lcrr}
\hline & HR & $95 \%$ CI & $p$ value \\
\hline Renal events & & & 0.023 \\
DR (yes vs. no) & 1.539 & $1.332-1.842$ & $<0.001$ \\
eGFR, mL/min/1.73 m² & 0.943 & $0.919-0.961$ & 0.048 \\
24-h proU, g & 1.211 & $1.132-1.387$ & 0.019 \\
Cardiovascular events & & & 0.038 \\
Age (10 years) & 1.672 & $1.487-1.821$ & 0.027 \\
HbA1C, \% & 1.398 & $1.197-1.876$ & 0.043 \\
24-h proU, g & 1.453 & $1.289-1.672$ & $1.001-1.052$ \\
Phosphorus, mmol/L & 1.021 & & \\
\hline
\end{tabular}

$p$ values $<0.05$ were considered to indicate a statistically significant difference. The following variables were incorporated as covariates: age, sex, BMI, course of DM, use of insulin, presence of diabetic retinopathy, hypertension, SBP, DBP, HbA1c, FBG, 2h-PPG, eGFR, 24-h proU, hemoglobin, serum albumin, calcium, phosphorus, TC, TG, HDL-C, and LDL-C. DR, diabetic retinopathy.

\section{Discussion}

Worldwide, almost 3\% of newly diagnosed patients with T2DM have overt nephropathy, and approximately $20-30 \%$ of diabetic patients will experience renal injury during their lifetime [1, 2]. However, the specific composition of renal diseases in diabetic patients remained obscure with the reported incidence of NDRD varying widely from 18.1 to $82.9 \%$ [3-12]. In our center, 179 patients (54.9\%) were diagnosed with DN, while 147 (45.1\%) had NDRD after the exclusion of patients with mixed lesions. A previous nationwide renal biopsy survey in China reported similar findings, with $44.7 \%$ of diabetic patients diagnosed as pure DN, $49.1 \%$ as NDRD alone, and 6.2\% as NDRD superimposed on DN [12]. Besides, the leading causes of NDRD also varied largely. For example, MN and IgAN were the two most common pathological NDRD diagnoses in Asian [5, 7, 12] and Croatian patients [8], which is consistent with our findings. However, focal segmental glomerulosclerosis is more prevalent in the USA and New Zealand [6,9], while acute interstitial nephritis is ranked first in NDRD in Malaysia and India [33,34]. Therefore, multicentered studies are indispensable to confirm the precise spectrum of renal diseases in diabetic patients with renal injury.

Given the invasiveness of renal biopsy, identification of non-invasive clinical predictors for DN may be extremely useful in clinical practice. Studies have confirmed the predictive value of DR for DN [35, 36]. DR and DN are both microvascular complications and similar mechanisms have been identified, such as oxidative stress and the formation of advanced glycation end-products [37]. Here, we confirmed that the presence of DR does indeed indicate the possibility of biopsy-proven DN. In addition, family history of diabetes and dependence on insulin were also identified as risk factors for DN. Therefore, close attention should be paid to patients with DR, family history of diabetes, and insulin therapy as they are more likely to develop DN.

CVD has remained the leading cause of death in patients with T1DM and T2DM [14]. Studies indicate that DN patients are more likely to suffer from severe CVD with higher morbidity and mortality versus those without DN [15-18, 38]. In the Go-DARTS and MESA studies, LVH was identified as adverse cardiac remodeling in patients with T2DM and renal insufficiency $[39,40]$. Mounting evidence also indicates the predictive value of LVH for cardiovascular events $[20,21]$. However, few studies have focused on the incidence and severity of 


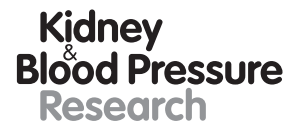

Research \begin{tabular}{l|l}
\hline Kidney Blood Press Res 2020;45:378-390 \\
\hline DOI: 10.1159/000505919 & $\begin{array}{l}\text { @ 2020 The Author(s). Published by S. Karger AG, Basel } \\
\text { www.karger.com/kbr }\end{array}$ \\
\hline
\end{tabular}

Wang et al.: Poor Prognosis of Biopsy-Proven Diabetic Nephropathy

LVH in diabetic patients with renal biopsy. In the current study, we found that LVH was present in about one-third of patients with biopsy-proven DN and this incidence was significantly higher than NDRD patients. Several pathophysiological changes may explain the high incidence of LVH in DN patients, including insulin resistance [19], decreased metabolic flexibility [41], increased accumulation of advanced glycation end-products [42], and activation of systemic RAAS [43]. Therefore, the detection of LVH is important and may serve as an early indicator of cardiovascular damage, especially in DN patients.

PAD is another common clinical manifestation in diabetic patients, and its incidence was shown to be comparable to that of coronary events and stroke according to the ADVANCE trial [44]. Studies have also established the association between PAD and an increased risk of cardiovascular morbidity and mortality [15]. However, most participants in previous studies were clinically diagnosed as DN without pathological confirmation, and differences in PAD between DN and NDRD had not previously been evaluated. In the current study, we for the first time demonstrated that PAD was more prevalent in biopsy-proven DN patients, who exhibited thicker carotid and femoral IMT, as well as more peripheral atherosclerotic plaques. Notably, plaques with low echogenicity in lower extremity arteries were also more prevalent in the DN group, indicating a higher risk of rupture in these patients. Given the numerous specific changes associated with DN, including insulin resistance, microvascular dysfunction, and deposition of calcium-phosphate products [18], these patients can be considered to be vulnerable of developing PAD, and timely management should be established to prevent its further progression.

In our study, comparison of renal and cardiovascular outcomes in the DN and NDRD groups demonstrated that DN patients tended to have poorer renal and cardiovascular survival than NDRD patients despite their similar renal function at the baseline, suggesting the reduction of eGFR is simply a marker of systemic vascular damage, and not its cause. The better renal prognosis associated with NDRD may be related to clinical stability or remission of certain diseases, such as IgAN and MN, which are potentially treatable with RAAS inhibitors or immunosuppressive therapy. Therefore, with the recovery and preservation of renal function in patients with NDRD, fewer cardiovascular risk factors such as water-sodium retention, hypertension, and anemia were present, resulting in a better cardiovascular prognosis than in the DN group. Therefore, proper management and early prevention is of great importance in reducing renal and cardiovascular events in patients with biopsy-proven DN.

The current study is the first to demonstrate that patients with DN are likely to experience more severe LVH and PAD than those with NDRD. However, the study has several potential concerns and limitations. First, this was a single-center, retrospective study and the selection bias was unavoidable. Therefore, multicentered and prospective studies are indispensable for further understanding the spectrum of biopsy-proven renal disease among diabetic patients. Secondly, we were not able to obtain comparable clinical information for diabetic patients with NDRD superimposed on DN due to the small sample size, which was also a weakness in many other studies. Due to the large variations of sample sizes, we were unable to perform further comparisons between DN patients with DR and those without DR regarding cardiac remodeling, atherosclerosis of carotid, and lower extremity arteries along with their renal and cardiovascular survival. Therefore, further studies with a proper sample size are necessary to provide a better view of the association between DR and biopsy-proven DN and further guide our clinical practice. Thirdly, we are still working on the relationship between the pathological classification of DN and clinical characteristics, as well as renal and cardiovascular survival, and hopefully we will be able to clarify their association with more participants being involved.

In conclusion, patients with biopsy-proven DN were more likely to develop LVH and PAD and had poorer renal and cardiovascular outcomes than NDRD patients. Assessment of renal 
pathology remains the gold standard for the diagnosis of kidney diseases, and it is essential that nephrologists understand the importance of renal biopsy for accurate diagnosis, appropriate treatment, and ultimately improved prognosis in diabetic patients with kidney injury.

\section{Acknowledgements}

The authors thank the patients for participation in our study and the staff of the Department of Nephrology at Shanghai Jiao Tong University Affiliated Sixth People's Hospital for assistance.

\section{Statement of Ethics}

The study protocol was approved by the Ethics Committee of the Shanghai Jiao Tong University Affiliated Sixth People's Hospital and was conducted in full accordance with the principles of the Declaration of Helsinki. All patients provided written informed consent.

\section{Disclosure Statement}

The authors have no conflicts of interest to declare.

\section{Funding Sources}

This study was supported by the National Nature Science Foundation of China (81670657, 81870504, 81870468, 81400735), National "Twelfth Five-Year" Plan for Science and Technology Support (2013BAI02B01-01), the Interdisciplinary Program of Shanghai Jiao Tong University (YG2017MS10), Shanghai Municipal Commission of Science and Technology (grant No.18DZ2260200), Open Project of Shanghai Key Laboratory of Sleep-Disordered Breathing (SHKSDB-KF-19-04), and Science and Technology Commission of Shanghai Municipality (14DZ2260200, the project of Shanghai Key Laboratory of Kidney and Blood Purification).

\section{Author Contributions}

In the study, Y.F., N.W., and Y.W. designed the research project. Y.W., T.Z., Qi.Z., Y.F., Z.L., S.L., and L.H. performed the study, collected the clinical data, and worked on the follow-up of patients. Y.W., Qu.Z., and Y.D. analyzed the data.Y.W. and T.Z. drafted the manuscript. Y.F. and N.W. revised and approved the final version of the manuscript.

\section{References}

1 Thomas MC, Cooper ME, Zimmet P. Changing epidemiology of type 2 diabetes mellitus and associated chronic kidney disease. Nat Rev Nephrol. 2016 Feb;12(2):73-81.

2 Zhang L, Long J, Jiang W, Shi Y, He X, Zhou Z, et al. Trends in Chronic Kidney Disease in China. N Engl J Med. 2016 Sep;375(9):905-6.

3 Mise K, Hoshino J, Ubara Y, Sumida K, Hiramatsu R, Hasegawa E, et al. Renal prognosis a long time after renal biopsy on patients with diabetic nephropathy. Nephrol Dial Transplant. 2014 Jan;29(1):109-18. 
Wang et al.: Poor Prognosis of Biopsy-Proven Diabetic Nephropathy

4 Pavkov ME, Knowler WC, Lemley KV, Mason CC, Myers BD, Nelson RG. Early renal function decline in type 2 diabetes. Clin J Am Soc Nephrol. 2012 Jan;7(1):78-84.

5 Li L, Zhang X, Li Z, Zhang R, Guo R, Yin Q, et al. Renal pathological implications in type 2 diabetes mellitus patients with renal involvement. J Diabetes Complications. 2017 Jan;31(1):114-21.

6 Sharma SG, Bomback AS, Radhakrishnan J, Herlitz LC, Stokes MB, Markowitz GS, et al. The modern spectrum of renal biopsy findings in patients with diabetes. Clin J Am Soc Nephrol. 2013 Oct;8(10):1718-24.

7 Oh SW, Kim S, Na KY, Chae DW, Kim S, Jin DC, et al. Clinical implications of pathologic diagnosis and classification for diabetic nephropathy. Diabetes Res Clin Pract. 2012 Sep;97(3):418-24.

8 Horvatic I, Tisljar M, Kacinari P, Matesic I, Bulimbasic S, Galesic Ljubanovic D, et al. Non-diabetic renal disease in Croatian patients with type 2 diabetes mellitus. Diabetes Res Clin Pract. 2014 Jun;104(3):443-50.

9 Zwi LJ, Yiu TS, Marshall MR, Lam-Po-Tang MK. Non-diabetic renal diseases in a multi-ethnic New Zealand cohort with type 2 diabetes mellitus: clinical and histopathological features. Pathology. 2014 Aug;46(5):42432.

10 Klessens CQ, Woutman TD, Veraar KA, Zandbergen M, Valk EJ, Rotmans JI, et al. An autopsy study suggests that diabetic nephropathy is underdiagnosed. Kidney Int. 2016 Jul;90(1):149-56.

11 An Y, Xu F, Le W, Ge Y, Zhou M, Chen H, et al. Renal histologic changes and the outcome in patients with diabetic nephropathy. Nephrol Dial Transplant. 2015 Feb;30(2):257-66.

12 Liu D, Huang T, Chen N, Xu G, Zhang P, Luo Y, et al. The modern spectrum of biopsy-proven renal disease in Chinese diabetic patients-a retrospective descriptive study. PeerJ. 2018 Mar;6:e4522.

13 Bhupathiraju SN, Hu FB. Epidemiology of Obesity and Diabetes and Their Cardiovascular Complications. Circ Res. 2016 May;118(11):1723-35.

14 Morrish NJ, Wang SL, Stevens LK, Fuller JH, Keen H. Mortality and causes of death in the WHO Multinational Study of Vascular Disease in Diabetes. Diabetologia. 2001 Sep;44(S2 Suppl 2):S14-21.

15 Mohammedi K, Woodward M, Hirakawa Y, Zoungas S, Williams B, Lisheng L, et al.; ADVANCE Collaborative Group. Micro-vascular and macrovascular disease and risk for major peripheral arterial disease in patients with type 2 diabetes. Diabetes Care. 2016 Oct;39(10):1796-803.

16 Hägg S, Thorn LM, Putaala J, Liebkind R, Harjutsalo V, Forsblom C M, et al. FinnDiane Study Group. Incidence of stroke according to presence of diabetic nephropathy and severe diabetic retinopathy in patients with type 1 diabetes. Diabetes Care 2013;36(12):4140-6

17 Barrios C, Pascual J, Otero S, Soler MJ, Rodríguez E, Collado S, et al.; investigators of the NEFRONA study Diabetic nephropathy is an independent factor associated to severe subclinical atheromatous disease. Atherosclerosis. 2015 Sep;242(1):37-44

18 Ninomiya T, Perkovic V, de Galan BE, Zoungas S, Pillai A, Jardine M, et al.; ADVANCE Collaborative Group. Albuminuria and kidney function independently predict cardiovascular and renal outcomes in diabetes. J Am Soc Nephrol. 2009 Aug;20(8):1813-21.

19 Jia G, DeMarco VG, Sowers JR. Insulin resistance and hyperinsulinaemia in diabetic cardiomyopathy. Nat Rev Endocrinol. 2016 Mar;12(3):144-53.

20 O'Neal WT, Almahmoud MF, Qureshi WT, Soliman EZ. Electrocardiographic and Echocardiographic Left Ventricular Hypertrophy in the Prediction of Stroke in the Elderly. J Stroke Cerebrovasc Dis. 2015 Sep;24(9): 1991-7.

21 Ang DS, Fahey TP, Wright GA, Struthers AD. Development and validation of a clinical score to identify echocardiographic left ventricular hypertrophy in patients with cardiovascular disease. Am J Hypertens. 2008 Sep; 21(9):1011-7.

22 O'Hare AM, Vittinghoff E, Hsia J, Shlipak MG. Renal insufficiency and the risk of lower extremity peripheral arterial disease: results from the Heart and Estrogen/Progestin Replacement Study (HERS). J Am Soc Nephrol. 2004 Apr;15(4):1046-51.

23 Mohammedi K, Woodward M, Hirakawa Y, Zoungas S, Colagiuri S, Hamet P, et al.; ADVANCE Collaborative Group. Presentations of major peripheral arterial disease and risk of major outcomes in patients with type 2 diabetes: results from the ADVANCE-ON study. Cardiovasc Diabetol. 2016 Sep;15(1):129-38.

24 Norman PE, Davis WA, Bruce DG, Davis TM. Peripheral arterial disease and risk of cardiac death in type 2 diabetes: the Fremantle Diabetes Study. Diabetes Care. 2006 Mar;29(3):575-80.

25 Dormandy JA, Betteridge DJ, Schernthaner G, Pirags V, Norgren L; PROactive investigators. Impact of peripheral arterial disease in patients with diabetes-results from PROactive (PROactive 11). Atherosclerosis. 2009 Jan; 202(1):272-81.

26 Tervaert TW, Mooyaart AL, Amann K, Cohen AH, Cook HT, Drachenberg CB, et al.; Renal Pathology Society. Pathologic classification of diabetic nephropathy. J Am Soc Nephrol. 2010 Apr;21(4):556-63.

27 Cheitlin MD, Armstrong WF, Aurigemma GP, Beller GA, Bierman FZ, Davis JL, et al.; American College of Cardiology; American Heart Association; American Society of Echocardiography. ACC/AHA/ASE 2003 guideline update for the clinical application of echocardiography: summary article: a report of the American College of Cardiology/American Heart Association Task Force on Practice Guidelines (ACC/AHA/ASE Committee to Update the 1997 Guidelines for the Clinical Application of Echocardiography). Circulation. 2003 Sep; 108(9): 1146-62.

28 Jafary FH. Devereux formula for left ventricular mass-be careful to use the right units of measurement. J Am Soc Echocardiogr. 2007 Jun;20(6):783. 
29 Verbraecken J, Van de Heyning P, De Backer W, Van Gaal L. Body surface area in normal-weight, overweight, and obese adults. A comparison study. Metabolism. 2006 Apr;55(4):515-24.

30 Lang RM, Bierig M, Devereux RB, Flachskampf FA, Foster E, Pellikka PA, et al.; Chamber Quantification Writing Group; American Society of Echocardiography's Guidelines and Standards Committee; European Association of Echocardiography. Recommendations for chamber quantification: a report from the American Society of Echocardiography's Guidelines and Standards Committee and the Chamber Quantification Writing Group, developed in conjunction with the European Association of Echocardiography, a branch of the European Society of Cardiology. J Am Soc Echocardiogr. 2005 Dec;18(12):1440-63.

31 Touboul PJ, Hennerici MG, Meairs S, Adams H, Amarenco P, Bornstein N, et al. Mannheim carotid intima-media thickness and plaque consensus (2004-2006-2011). Cerebrovasc Dis. 2012;34(4):290-6.

32 Hicks KA, Tcheng JE, Bozkurt B, Chaitman BR, Cutlip DE, Farb A, et al.; American College of Cardiology; American Heart Association. 2014 ACC/AHA Key Data Elements and Definitions for Cardiovascular Endpoint Events in Clinical Trials: A Report of the American College of Cardiology/American Heart Association Task Force on Clinical Data Standards (Writing Committee to Develop Cardiovascular Endpoints Data Standards). Circulation. 2015 Jul;132(4):302-61.

33 Soni SS, Gowrishankar S, Kishan AG, Raman A. Non diabetic renal disease in type 2 diabetes mellitus. Nephrology (Carlton). 2006 Dec;11(6):533-7.

34 Chong YB, Keng TC, Tan LP, Ng KP, Kong WY, Wong CM, et al. Clinical predictors of non-diabetic renal disease and role of renal biopsy in diabetic patients with renal involvement: a single centre review. Ren Fail. 2012; 34(3):323-8.

35 Kramer CK, Retnakaran R. Concordance of retinopathy and nephropathy over time in Type 1 diabetes: an analysis of data from the Diabetes Control and Complications Trial. Diabet Med. 2013 Nov;30(11):1333-41.

36 Klein R, Zinman B, Gardiner R, Suissa S, Donnelly SM, Sinaiko AR, et al.; Renin-Angiotensin System Study. The relationship of diabetic retinopathy to preclinical diabetic glomerulopathy lesions in type 1 diabetic patients: the Renin-Angiotensin System Study. Diabetes. 2005 Feb;54(2):527-33.

37 Sheetz MJ, King GL. Molecular understanding of hyperglycemia's adverse effects for diabetic complications. JAMA. 2002 Nov;288(20):2579-88.

38 Sarafidis PA, Bakris GL. Cardiovascular disease in CKD in 2014: new insights into cardiovascular risk factors and outcomes. Nat Rev Nephrol. 2015 Feb;11(2):70-2.

39 Moran A, Katz R, Jenny NS, Astor B, Bluemke DA, Lima JA, et al. Left ventricular hypertrophy in mild and moderate reduction in kidney function determined using cardiac magnetic resonance imaging and cystatin C: the multi-ethnic study of atherosclerosis (MESA). Am J Kidney Dis. 2008 Nov;52(5):839-48.

40 Parry HM, Donnelly LA, Van Zuydam N, Doney AS, Elder DH, Morris AD, et al.; Wellcome Trust Case Control Consortium 2. Genetic variants predicting left ventricular hypertrophy in a diabetic population: a Go-DARTS study including meta-analysis [J]. Cardiovasc Diabetol. 2013 Jul;12(1):109-19.

41 Guo CA, Guo S. Insulin receptor substrate signaling controls cardiac energy metabolism and heart failure. J Endocrinol. 2017 Jun;233(3):R131-43.

42 Willemsen S, Hartog JW, van Veldhuisen DJ, van der Meer P, Roze JF, Jaarsma T, et al. The role of advanced glycation end-products and their receptor on outcome in heart failure patients with preserved and reduced ejection fraction. Am Heart J. 2012 Nov;164(5):742-749.e3.

43 Baudrand R, Gupta N, Garza AE, Vaidya A, Leopold JA, Hopkins PN, et al. Caveolin 1 Modulates AldosteroneMediated Pathways of Glucose and Lipid Homeostasis. J Am Heart Assoc. 2016 Sep;5(10):e003845.

44 Patel A, MacMahon S, Chalmers J, Neal B, Billot L, Woodward M, et al.; ADVANCE Collaborative Group. Intensive blood glucose control and vascular outcomes in patients with type 2 diabetes. N Engl J Med. 2008 Jun;358(24): 2560-72. 